\title{
A Realist Review of the Home care Literature and its Blind Spots
}

\author{
Damien Contandriopoulos ${ }^{1}$, Kelli Stajduhar ${ }^{1}$, Tanya Sanders ${ }^{1}$, Annie Carrier ${ }^{2}$, Ami \\ Bitschy $^{3}$, and Laura Funk ${ }^{4}$ \\ ${ }^{1}$ University of Victoria \\ ${ }^{2}$ Université de Sherbrooke \\ ${ }^{3}$ University of Victoria Institute on Aging \& Lifelong Health \\ ${ }^{4}$ University of Manitoba
}

July 18, 2021

\begin{abstract}
Rationale, aims and objectives: There is a large body of literature from all over the world that describes, analyzes, or evaluates home care models and interventions. The present article aims to identify the practical lessons that can be gained from a systematic examination of that literature. Method: We conducted a three-step sequential search process from which 113 documents were selected. That corpus was then narratively analyzed according to a realist review approach. Results: A first level of observation is that there are multiple blind spots in the existing literature on home care. The definition and delimitation of what constitutes home care services is generally under-discussed. In the same way, the composition of the basket of care provided and its fit with the need of recipients is under-addressed. Finally, the literature relies heavily on RCTs whose practical contribution to decisions or policy is disputable.At a second level, our analysis suggests that three mechanisms (system integration, case management and relational continuity) are core characteristics of home care models effectiveness. Conclusion: We conclude by providing advice for creating effective home care system change through rational and participatory design and evaluation alongside public dialogue about the purpose and future of home care.
\end{abstract}

Realist Review of the Home care Literature

Damien Contandriopoulos ${ }^{1,2}$, Kelli Stajduhar ${ }^{1,2}$, Tanya Sanders ${ }^{1,3}$, Annie Carrier ${ }^{4,5}$ Ami Bitschy ${ }^{2}$, Laura Funk $^{6}$

1 School of Nursing, University of Victoria (Victoria, British-Columbia, Canada) 2 Institute on Aging and Lifelong Health, University of Victoria (Victoria, British-Columbia, Canada) 3 School of Nursing, Thompson Rivers University (Kamloops, British Columbia, Canada) 4 École de Réadaptation, Faculté de Médecine et des sciences de la santé, Université de Sherbrooke (Sherbrooke, Québec, Canada) 5 Centre de recherche sur le vieillissement, Centre intégré universitaire de santé et de services sociaux de l'Estrie - Centre hospitalier universitaire de Sherbrooke (Sherbrooke, Québec, Canada) 6 Department of Sociology and Criminology, University of Manitoba (Winnipeg, Manitoba, Canada)

Correspondence to:

Damien Contandriopoulos School of Nursing University of Victoria PO Box 1700 STN CSC Victoria BC V8W 2Y2, Canada 


\begin{abstract}
Rationale, aims and objectives: There is a large body of literature from all over the world that describes, analyzes, or evaluates home care models and interventions. The present article aims to identify the practical lessons that can be gained from a systematic examination of that literature. Method: We conducted a threestep sequential search process from which 113 documents were selected. That corpus was then narratively analyzed according to a realist review approach. Results: A first level of observation is that there are multiple blind spots in the existing literature on home care. The definition and delimitation of what constitutes home care services is generally under-discussed. In the same way, the composition of the basket of care provided and its fit with the need of recipients is under-addressed. Finally, the literature relies heavily on RCTs whose practical contribution to decisions or policy is disputable.At a second level, our analysis suggests that three mechanisms (system integration, case management and relational continuity) are core characteristics of home care models effectiveness. Conclusion: We conclude by providing advice for creating effective home care system change through rational and participatory design and evaluation alongside public dialogue about the purpose and future of home care.
\end{abstract}

Keywords:

Home Care Services, Aging, Policy, Case Management, Systems Integration, Nursing, Occupational Therapists

\title{
Introduction
}

For decades now, the provision of effective and accessible home care services for older adults has been touted as a solution to the challenges posed by aging populations. However, in light of the recent hecatomb of COVID-19 deaths in long-term care institutions, the importance and urgency of optimizing home care services is stronger than ever.

In this context, we conducted a large scale and broadly focused, realist-inspired narrative review aimed at answering three questions. First, what is home care? Though the term is commonly used as if the definition was self-evident, the nature, boundaries and objectives of home care vary a lot depending on jurisdictions and authors. Second, what evidence exists on the effectiveness of home care models and interventions? Many intervention-specific systematic reviews have been published but few policy-level efforts exist that integrate insights into the links between home care models and their outcomes. Third, what is known about the causal processes involved in the production of those effects? This last question aims at summarizing available evidence to inform intervention design, optimization and evaluation. In conclusion, our analysis suggests that three mechanisms (system integration, case management and relational continuity) are core characteristics of home care models' effectiveness.

\section{Methods}

Some systematic review approaches, such as those produced by the Cochrane collaboration, aim at exhaustively summarizing the evidence about the effects of a given intervention. The realist review approach differs in that identifying the effects of interventions is not the end result so much as a step toward understanding the causal processes involved in the production of those effects ${ }^{1-3}$. This is similar to what the field of evaluation describes as reverse logic analysis where the aim is to identify the causal links between characteristics of given interventions and their outcomes in order to provide insights on how to produce similar outcomes in the design of new interventions ${ }^{4}$. We initially expected to be able to conduct a detailed reverse logic analysis based on the available scientific literature documenting home care delivery models. However, the literature identified provided too few insights on the causal processes involved to allow us to go beyond the identification of three main characteristics of promising interventions. 


\section{Search Method}

To maximize the breadth of the search, we relied on three different, sequential, search approaches. The starting point was a keyword-based search in MEDLINE and CINAHL conducted in June 2019. This search led to the identification of 1628 non-duplicate references that were reviewed independently by two reviewers on the basis of title and abstract. Two criteria were used. First, the document had to provide relevant information on the delivery of case managed, integrated or consumer-directed home and community services. Home and community services could include but could not be limited exclusively to medical care. Second, the population receiving the care needed to be community dwelling, with either a majority aged 65 years and over, or with a subsample of persons aged 65 and over for whom results were reported separately. Among the references identified, 107 were selected for full-text analysis. The full text was then independently appraised by two reviewers. 35 articles were selected at the end of the first step.

For the second step, the bibliographies of the 35 papers previously selected were compiled and reviewed to identify potentially relevant titles. This led to the identification of 94 new references that were then reviewed according to the same double-blind processes used in the first step. Of those, 50 documents were selected for full-text review and 34 included in the analysis. We also included one paper independently identified by a co-author. At the end of the second step, 70 documents were identified.

The third step was a reverse search in MEDLINE for all articles citing at least one of the 70 documents identified through the previous two steps. This led to the identification of 1102 non-duplicate references. Of those, 71 had already been reviewed previously (recaptures). The remaining 1034 were processed in the same way as described previously. Of those, 78 were deemed appropriate for a full-text review and 42 were retained. At this stage, a second paper provided by a co-author was also added. The low number of recaptures suggests that the total number of articles that fit our focus of interest is likely very large ${ }^{5}$.

In the end, 113 documents were included in the analysis11The complete list can be accessed as a PubMed bibliography at https://www.ncbi.nlm.nih.gov/myncbi/1Dm-PibJgyqcPF/bibliography/public/.. Figure 1 (below) provides a flowchart for the process.

[Insert figure 1: Search process flowchart]

\section{Relevance and strength appraisal}

Our approach to full-text appraisal relied on two scores: one for methodological quality (strong=3, acceptable $=2$, weak=1) and one for relevance (highly relevant $=3$, some relevant elements $=2$, not relevant $=1$ ). Documents were only selected for inclusion at this next stage if they had a combined score of 3 or above. The inclusion threshold was deliberately set low enough to maximize the sensitivity of the search. Divergences in scoring were resolved by discussion and consensus was reached in all cases.

As this system relies on a moving threshold regarding the strength of the study design, some additional discussion might be warranted. The type of review we conducted is integrative and iterative in nature. First, it is integrative, as different types of evidence (description, typologies, outcome evaluations, etc.) are brought together with the aim to identify desirable characteristics of home care delivery models. Second, it is iterative, as the focus of the review was refined on an ongoing basis as it progressed. Therefore, some of the documents included in the analysis offer robust evidence while others are descriptive or rely on weak study designs. However, when analyzing the data itself, the strength of the evidence was taken into account in a contextualized way. For example, study design matters when analyzing potential links between interventions and outcomes. But study design matters much less than face validity when assessing the usefulness of a typology of home care delivery models. 


\section{Data organization and coding}

The documents were coded on an ongoing basis throughout the three phases according to a modified PICO grid. The main modification to the PICO format was that the "C" here refers to the causality presumptions made in the paper (what underlying hypotheses are made or implied linking the intervention to the expected outcomes?). The other items were usual elements of the PICO format, including: Population (Who is receiving the services? For example health issue, age, insurance status, location, etc.); Intervention (What services are being offered? Professionals involved, intensity, duration, etc.); and Outcomes (what outcomes are described or measured?). We also coded articles by country and type of method. When relevant, additional information such as formal definitions of home care were retrieved during coding. Also, some documents included in the analysis would not easily be classified within this coding grid (for example, broadly focused reviews of the literature) and those where coded on an ad hoc basis. The coding results for each article were 164 words long on average (standard deviation 68 words), for a total of 18585 words. The analysis also heavily relied on multiple iterative reading of the full text of each document.

\section{Results}

\section{Description of the articles}

The documents reviewed span almost three decades (1992 to 2020) and provide information on home care services or interventions in 16 countries. Among the 113 documents reviewed, 27 were secondary reviews of the evidence, including 17 systematic reviews. The remaining 87 documents included 24 articles reporting the results of 22 randomized controlled trials (RCT), 31 documents based on non-RCT quantitative methods, 11 documents based on qualitative methods, 2 documents relying on mixed methods and 16 documents providing purely descriptive or conceptual content.

\section{Definition, Types and Objectives of Home Care Services}

The implicit definition of home care in the studies reviewed here appears to be any broadly defined healthrelated services provided in people's homes, as well as the remote coordination of care provided to the recipient of such services ${ }^{6-8}$. Content-wise, home care can include a vast array of services ranging from specialized medicine (for example, care provided by geriatricians, oncologists, psychiatrists, etc.) to services to help with daily living (meal preparation, house cleaning, etc.) and various types of care designed to promote health, gain or maintain autonomy, or cure ailments ${ }^{9}$. The diversity and breadth of what is described as home care in the scientific literature constitutes a significant challenge for integrative reviews such as ours.

Beyond that general definition (or lack thereof), the literature we analyzed can be characterized based on two main splits that we identified. First, there is a clear divide between fixed duration "reablement" interventions (also named restorative care in some jurisdictions) and indefinite-duration need-based interventions. The underlying causal hypothesis of reablement is that an intensive, multidisciplinary, time-limited (generally under 12 weeks) service can improve an older adult's independence in daily activities so that no further services are needed afterwards ${ }^{10-12}$. Such an approach has some good face value for specific groups (postdischarge patients for example). However, the literature analyzed often treats reablement as a substitute for indefinite-duration home care focused on supporting people whose functional independence is likely on a downward trajectory. For example, articles focused on assessing the effectiveness of reablement interventions tend to compare the outcomes of such interventions with "regular" long-term home care services" ${ }^{10-17}$.

The second split has to do with the objectives of home care programs or interventions we reviewed ${ }^{6}$. The first type of objectives are individual in focus and focus on the autonomy, satisfaction, and health of the older adults or their family/friend caregivers. Such objectives are frequently assessed using standardized instruments focused on health status (i.e. SF-12 and SF-36), mental health (i.e. 11-item CES-D Depression Scale, SPMSQ, Kessler-10 anxiety and depression scale), quality of life (i.e. EUROQOL, WHOQOL-Bref), functional capacities (i.e. OARS-IADL, Groningen Activity Restriction Scale-3, Barthel Index of Activities of 
Daily Living), caregivers' burden and perceived unmet needs, or idiosyncratic indicators linked with specific interventions. The second type of objectives are societal in focus and focus on the system-level efficiency, appropriateness of resource allocation and the coherence between social values and available services. Indicators of system level objectives focus more on endpoint events (such as death or admission to hospital or nursing home), service use intensity measured through administrative databases (i.e. emergency room visits, number of hospitalizations, number of days hospitalized, number of visits, continued use of home care after a given time or number of visits), cost or cost benefits from those interventions as well as broader policy-level discussion of the alignment between practices and values.

\section{Assessing Home Care Effectiveness}

\section{Objectives and indicators}

Among the 113 reviewed papers, 45 were focused on measuring or synthesizing the effectiveness of one or multiple home care interventions. Our first challenge when trying to synthesize the evidence on home care intervention effectiveness revolved around the vague operational definition of the concept of home care itself, and the diversity in the objectives being pursued ${ }^{9}$. The effectiveness-focused articles we reviewed each relied on given sets of indicators. Notably however, the rationale for those indicators were seldom discussed in relation to the ultimate goal of the intervention itself. For example, focusing on recipients' quality of life versus focusing on rates of hospitalization or number of hospital visits suggest significantly different underlying objectives.

\section{Shifting Goalposts}

A second challenge concerns the research designs employed in the research. Most of the effectiveness-focused studies rely on randomized controlled trials (RCTs) and systematic reviews thereof. However, the nature of home care interventions makes it difficult to appropriately deploy such study designs. The vast majority of the RCTs we reviewed compare a given home care intervention to "usual care" but fail to define that baseline of "usual care." In some studies, the "usual care" provided consisted of a generous basket of services, while in others it amounted to very little. Yet we only found three studies, all from the United States, comparing the outcomes of providing versus not providing home care services ${ }^{18-20}$. Those studies all showed clear benefits from receiving home care services. There are obvious ethical considerations at play that might explain why few studies explicitly compare the outcome of providing versus not providing services to the same population. However, beyond those three studies, when looking at the whole field it is important to contrast, on the one hand, the significant efforts and resources invested in running RCTs measuring the precise level of marginal benefits between two models of home care and, on the other hand, the relative lack of interest in demonstrating the gain of providing home care in itself.

\section{Unclear Causal Processes}

Compounding the problem, most evaluations of a given home care intervention provided very little, if any, details on the nature of that intervention ${ }^{17}$. The descriptions generally mentioned some elements, such as numbers of home visits, intervention durations, or types of professionals involved, but often failed to provide the level of detail needed to understand the process involved in the production of the outcomes being measured. This observation was also noted in other reviews of the field ${ }^{21,22}$.

Because of the limitations discussed above, it is generally impossible to know whether an intervention which outperformed the baseline in a given context might prove beneficial in another. It also makes it impossible to determine the relative effectiveness of interventions with similar objectives. More generally, the policy value of most of the effectiveness studies we reviewed were limited to the time and jurisdiction of when and where the study took place. 


\section{If It Were Easy, It Would Succeed More Often}

One observation that stands out from the quantitative evaluations of home care interventions we reviewed is this - designing an effective intervention is not easy. Of the 45 evaluations of home care effectiveness, 22 were able to demonstrate the success of a given home care model or intervention while 23 offered unconvincing or negative results. Other reviews in the field reported similar results ${ }^{23}$.

For example, Bouman, van Rossum, Nelemans, et al. ${ }^{24}$ systematic review of seven RCTs on preventive home visits found that those programs had no effect. Berger, Escher, Mengle, et al. ${ }^{25}$ systematic review of 38 articles on occupational therapy-based home care services found some benefits for participants' occupational performance, mixed results for quality-of-life indicators and no effect on health care utilization. Our analysis also included one primary study ${ }^{26}$ covering similar ground that found no meaningful effect from the restorative occupational therapy intervention studied.

Turning to reablement-focused interventions in particular, one 2013 Cochrane systematic review of this approach ${ }^{10}$ failed to identify any high quality evidence showing that such interventions worked. The literature we reviewed included one primary $\mathrm{RCT}^{13}$ that found a reablement intervention to be superior to regular care, yet others ${ }^{27,28}$ which found no meaningful benefits. Moreover, the primary studies of reablement services we reviewed $(n=10)$ tended to rely on methodological choices - such as outcome measures focused on short-term physical autonomy gains - that likely overemphasize the benefits of such approaches ${ }^{12}$.

Luker, Worley, Stanley, et al. ${ }^{29}$ conducted a systematic review of 31 RCTs of home care interventions aimed at preventing the institutionalization of older adults, and found no meaningful benefit overall. The authors then conducted a sub-analysis of what they described as "complex multifactorial interventions" which suggested that this subgroup of interventions do in fact lower institutionalization. However, the definition and boundaries of that subgroup of intereventions is unclear and this approach could be interpreted as data dredging (data dredging describe the aggressive slicing of data and use of sub-group analysis to find statistically significant results). Four of the studies labelled by Luker, Worley, Stanley, et al. ${ }^{29}$ as "complex multifactorial interventions" are part of our own data set. Two of those $\mathrm{e}^{30,31}$ found no meaningful effects and two $^{32,33}$ showed positive effects. Other primary RCTs reviewed here ${ }^{34}$ that focused explicitly on multifactorial interventions found no effect as compared to the control group. Aggressive sub-analysis and potential data dredging is also present in some primary studies focused on similar interventions ${ }^{35-37}$

We also reviewed various nurse-led assessment and coordination of care interventions in different high-risk populations ${ }^{38-40}$ that failed to measure a meaningful effect. One study combining three different RCTs ${ }^{33}$ found positive impacts on mental health in one study and no impact in the two other RCTs. Another study ${ }^{41}$ of nurse-based home care found some effects on secondary variables (such as the incidence of falls) but no improvement in the primary outcome measure, which was related to quality of life.

The RCTs we reviewed with interventions aimed at improving post discharge coordination between hospitals and home care services or providing additional home care post discharge for frail elderly patients both failed to measure any meaningful effects ${ }^{30,42}$.

Low, Fletcher, Gresham, et al. ${ }^{43}$ found no convincing link between the need for home care services, the wait time required to obtain said services, or even the amount of services allocated. They also found no link between the services received and outcomes measured, such as quality of life indicators.

This list of negative results provides half the portrait; as we stated earlier, nearly half of the effectivenessfocused literature we reviewed found significantly better outcomes for the intervention under study. What we want to emphasize with this summary of non-effects is that one should not underestimate the challenges related to the design and implementation of an effective and efficient home care intervention.

\section{Insights on What Seems to Work}

Three characteristics of home care interventions stand out in the subsample of research studies which found positive results: intersectoral integration, care coordination and relational continuity. Regarding integration, 
Bernabei, Landi, Gambassi, et al. ${ }^{44}$ RCT of a multidisciplinary, integrated social and health care intervention, among frail older adults in northern Italy, found it improved functionality and mental health and lowered hospital or nursing home admission and costs per recipient. Likewise, Landi, Gambassi, Pola, et al. ${ }^{45}$ quasi-experimental study on the effectiveness of another multidisciplinary, integrated social and health care intervention (also in in northern Italy), found it lowered hospital admissions and costs. Beland and colleagues ${ }^{32,46}$ led the development and pilot testing of SIPA, an integrated care intervention for vulnerable community-dwelling older adults in Québec (Canada). The SIPA model involves community-based multidisciplinary teams responsible for the full spectrum of care provision, and led to improved accessibility, satisfaction and lower per-recipient costs. Stewart, Georgiou, Westbrook ${ }^{47}$ systematic summary of the results from the broader PRISMA research program, a different model also from Québec (Canada), identified 45 journal articles and two books authored or co-authored by the team that, overall, support the effectiveness of integrated models of home care on client satisfaction and limiting functional decline.

In the same way, multiple studies on the effectiveness of care coordination in home care showed positive results. Onder, Liperoti, Soldato, et al. ${ }^{48}$ large-scale retrospective cohort study of multiple European case-management programs found those to effectively lower nursing home admissions. Scharlach, Graham, Berridge ${ }^{19}$ evaluation of an integrated assessment and care coordination model suggests an improvement in clients' satisfaction and autonomy. Markle-Reid, Weir, Browne, et al. ${ }^{49} \mathrm{RCT}$ on the benefit of proactively providing older people with home care nursing, health promotion and care coordination in Ontario (Canada), found positive effects on mental health for similar costs. Melis, van Eijken, Teerenstra, et al. ${ }^{9}$ ran a RCT in the Netherlands demonstrating improved client mental health associated with a nurse-centred home care assessment and management intervention. Morales-Asencio, Gonzalo-Jimenez, Martin-Santos, et al. 50 Spanish, quasi-experimental study showed that their nurse-led case management intervention had a positive impact on functional capacity and caregiver burden. Bass, Judge, Lynn Snow, et al. ${ }^{51}$ surmised that in the United States, a Veteran Health Administration's care-coordination program focused on caregiver needs lowered their level of unmet needs, strain and depression. Parsons, Senior, Kerse, et al. ${ }^{21}$ 's meta-analysis of RCTs of three different care management models in New Zealand highlighted an association between those models and lower long-term care admissions and the incidence of death. Lastly, Shapiro, Taylor ${ }^{18}$ 's USbased RCT of a nurse-led assessment and case-management intervention also found improved satisfaction, lowered institutionalization and deaths.

The third characteristic we want to discuss here is relational continuity. Relational continuity is an ongoing therapeutic relationship between a service recipient and a professional ${ }^{52}$. In comparison with integration and care coordination, there is far less hard evidence that links continuity with positive outcomes in the documents we reviewed. Three effectiveness-focused studies identified continuity as a characteristic associated with desirable outcomes ${ }^{49,53,54}$ while two other more analytical articles ${ }^{55,56}$ discuss its importance in high-performing home care models. However, 34 of the documents we reviewed (30\%) mention continuity - most often relational continuity, with informational continuity as a more secondary focus - as a desirable feature of home care interventions and a core process leading to high quality services.

The next section on the main insights and policy-level lessons that can be learned from the 113 documents analyzed here.

\section{Discussion}

\section{Something is (likely) better than nothing}

The first element to emphasize here is that the literature analyzed rarely if ever addresses the question of whether home care is beneficial in, and of, itself (and for what purpose). As noted, we only found three studies (all from the US) that compared the provision of some home care services to receiving no services at all. Unsurprisingly, they all conclude that some is better than none when it comes to home care. 
As per the hierarchies of evidence in the canons of medical research, the bulk of the research evaluating home care effectiveness relies on comparative study designs, primarily RCTs. The strength of well-conducted RCTs is to effectively control for many biases. However, what we found is that, at the macro-level, the over-reliance on RCTs and other comparative study designs in home care research have left core questions unaddressed. For example, we did not find any evidence allowing us to establish what services should be prioritized as core components of a home care basket of services.

What we found, instead, is that in most jurisdictions the basket of available home care services appears to have evolved somewhat haphazardly over long periods of time as a mix of public, non-owned ${ }^{57}$ and private services aimed at meeting the basic needs of some older people in the population. What needs are being addressed (and which are ignored), which populations are covered (or not) and what structures the accessibility of those services (capacity to pay, poverty, age, location, etc.) does not seem to follow any obvious pattern. It is perplexing that the core question of "what services should be offered in priority" is left mostly unaddressed by the literature we reviewed (with some exceptions ${ }^{7,58,59}$ ). It also raise ethical issues regarding distributive justice ${ }^{60}$.

Despite this blind spot in the literature, based on the effectiveness data reviewed as well as some of the qualitative evidence analyzed ${ }^{6,61,62}$ and on the basis of its face value, we have little doubt that home care provision is beneficial in itself. The next sections expand to the systematic analysis of mechanisms associated with effective home care in the entire body of 113 articles; here, we more fully elaborate the three interconnected elements that appear to be central in the design of effective and efficient home care interventions and service delivery models - system-level integration, case coordination/management, and relational continuity.

\section{System Level Integration}

The first characteristic is macro-systemic. Systems that achieve more integration seem more likely to produce desirable outcomes. Two levels of integration can be identified in the literature we analyzed. The first is the clinical integration of different types of health care services and the other is the inter-sectorial integration of broader social services and home care. The most common examples of clinical integration in our data are processes that connect acute hospital services and post-discharge home care services as well as ongoing collaboration between primary care physicians and home care teams. In our data, intersectoral integration is generally described at the micro-level of care-coordination or multidisciplinary teams. Many studies suggest that integration is a core element to design high-performing home care models ${ }^{32,44-47,63}$, but the evidence is not unambiguous and some have found the opposite ${ }^{64,65}$.

Since a small body of highly influential seminal work in the $1990 \mathrm{~s}^{66,67}$ there has been an abundance of literature on integration of care and systems ${ }^{68}$ the subtleties of which are beyond the scope of this paper to discuss. However, based on our analyses, home care models need both effective care integration and intersectoral integration. The recipients of home care services are often frail people with multiple needs. They will often enter and leave acute care settings, consult with primary care and specialist physicians as well as need housing-related support and other types of social services. The literature we reviewed suggests that there are significant benefits in efforts towards integration. However, the "how" part is left underaddressed.

\section{Care Coordination and Case Management}

The second mechanism that characterizes many of the successful interventions we reviewed was case management and coordination. Although there is some overlap in the concepts of integration and case management, we treat case management separately for two reasons. First, it is generally treated as such in the description of the home care models we reviewed. Second, the processes involved in case management are highly micro-individual and practical in nature. Case management is generally described as involving a sequential set of activities (i.e. assessment, development of individualized plans, connections with relevant service providers, problem solving and reassessment ${ }^{9,18,19,32,44,45,47,48,50,51}$ ). As noted earlier, many studies on home 
care interventions where case management and care coordination played a central role resulted in positive outcomes $^{9,18,19,28,48-51}$ although a few did not ${ }^{38,39}$.

Most of the home care case management roles we documented in the research were held by nurses. The data we analyzed does not allow for an in-depth analysis of the way case management produces its results. However, we hypothesize that the use of formal standardized assessment tools does not play a large role in improving outcomes. What seems more influential is the ease of coordination and the ability to problemsolve. We also hypothesize a significant dose-response relationship. Case management will be most effective when the caseload of case managers is kept to a level that allows them to properly do their work.

\section{Relational Continuity}

The last mechanism we want to discuss is relational continuity. Most of the literature we reviewed is written using role-based terms (the doctor, the nurse, the care coordinator, the occupational therapist) often without specifying how much, if any, relational continuity exists between these individuals and the client or family. One exception is a study by Russell, Rosati, Rosenfeld, et al. ${ }^{54}$ which directly focused on this element. It found that the level of relational continuity varied considerably between recipients of home care services and that it was strongly correlated with functional capacity and risk of hospitalization. Other studies that took relational continuity into consideration found converging results ${ }^{69}$. Conversely, studies of home care models with very low relational continuity ${ }^{70,71}$ identified this feature as a weakness. For example, a study by Gray, Sedhom ${ }^{71}$ suggests that the US approach to cluster care leads to lower satisfaction because segmenting client needs leads to very low relational continuity. Finally, some authors ${ }^{72}$ conceive of the relational nature of the work involved in home care provision as a defining characteristic of home care itself ${ }^{62,71,72}$. Overall, our results follow the same path. Home care provision is acutely relational, low-tech and dependent on the quality of the human and social relations involved. There are specific challenges related to establishing system-level home care delivery parameters that take this reality into account. In primary care, relational continuity has been shown to affect mortality rates $^{73,74}$ and we believe there are plausible indications it strongly influences the quality of the care provided and their outcomes in the home care context too.

\section{Conclusion}

This review was rooted in a significant effort to identify the existing literature so that three questions could be answered: What is home care, what is the evidence on home care intervention effectiveness and what is known about the causal processes involved. We eventually selected 113 documents that provided some answers to those questions. However, one key take home message from our analysis is that home care evidence base still contains significant blind spots. In particular, this research only provides very limited practical or policy-oriented guidance on how to improve or strengthen home care delivery. This hampers policymakers and advocacy groups in their efforts to create effective system change.

There are numerous specific "narrow" services that can be provided for older adults at home which can provide desirable outcomes for recipients. But the systemic benefit will not be optimized by randomly implementing some interventions based on local idiosyncratic parameters. Rationally designing a home care delivery model implies a series of sequential steps. The first would be to identify what system-level goals the model should achieve and the populations it should serve. The second step would be to identify what type of services are likely to achieve those goals in order to establish a basket of services. Finally would come the task of identifying the best ways and specific means to effectively and efficiently provide those services. We want also to stress two important elements. First, at each step, such mechanisms need to provide enough flexibility and local contextualization to escape the limitations of top-down, wall-to-wall approaches while ensuring equitable coverage and service provision. Second, we want to point-out that questions such as optimal team composition, funding model and administrative structures can only be meaningfully discussed at the third step of the process. 
These same three steps can also be used to establish an evaluative framework to appraise current and future home care models. First, what are the goals being pursued and populations being served? Are those choices aligned with societal values and system-level objectives? Second, what basket of services is being provided? Is the basket coherent with the goals put forward? Is the basket comprehensive enough, and how would we know? Third, what delivery approaches and interventions are used? Are these the most effective and efficient given available evidence? It should be noted that the first two questions are mostly value-driven and focused on the coherence of choices while the third is predominantly evidence-driven.

In conclusion, it could be noted that $30 \%$ of the documents we reviewed explicitly stated that the aging population will require stronger and better home care models, while another $18 \%$ mentioned unmet needs that home care could address. However, much of the knowledge needed to implement stronger, better home care is not provided by the literature. Future research on home care would benefit from more directly involving decision-makers and advocates in collaborative approaches, to more fully ensure that evidence is policy-relevant and that policymakers are not only attuned to methodological issues but are educated in the importance of public dialogue around the purpose and future of home care. National and international leadership and consensus-building are needed just as much in policy as research as we move forward to support older adults who live at home across the globe.

\section{Bibliography}

1. Pawson R. Evidence-based policy: the promise of 'realist synthesis'.Evaluation. 2002;8(3):340-358.

2. Pawson R. Evidence-based policy: a realist perpective. London: SAGE Publications; 2006.

3. Pawson R, Greenhalgh T, Harvey G, Walshe K. Realist review-a new method of systematic review designed for complex policy interventions.J Health Serv Res Policy. 2005;10(Suppl. 1):21-34.

4. Brousselle A, Champagne F. Program theory evaluation: logic analysisEval Prog Plan. 2011;34(1):69-78.

5. Seber GAF. Estimation of Animal Abundance. New York: MacMillan; 1982.

6. Thome B, Dykes A-K, Hallberg IR. Home care with regard to definition, care recipients, content and outcome: systematic literature review. Journal of Clinical Nursing. 2003;12:860-872.

7. MacAdam M. Home care: it's time for a Canadian model.Healthcarepapers. 2000;1(4):9-36.

8. Canada H. Home and community health care. Government of Canada,. https://www.canada.ca/en/health-canada/services/home-continuing-care/home-community-care.html.

Published 2016. Accessed April 29, 2021.

9. Melis RJF, van Eijken MIJ, Teerenstra S, et al. A randomized study of a multidisciplinary program to intervene on geriatric syndromes in vulnerable older people who live at home (Dutch EASYcare Study). The Journals Of Gerontology Series A, Biological Sciences And Medical Sciences. 2008;63(3):283-290.

10. Cochrane A, S. M, M. F, Molloy DW, Stevenson M, Donnelly M. Home-care 're-ablement' services for maintaining and improving older adults' functional independence. Cochrane Database of Systematic Reviews. 2013(11).

11. Ryburn B, Wells Y, Foreman P. Enabling independence: restorative approaches to home care provision for frail older adults. Health Soc Care Community. 2009;17(3):225-234.

12. Tuntland H, Aaslund MK, Espehaug B, Forland O, Kjeken I. Reablement in community-dwelling older adults: a randomised controlled trial.BMC Geriatrics. 2015;15:145-145.

13. Langeland E, Tuntland H, Folkestad B, Forland O, Jacobsen FF, Kjeken I. A multicenter investigation of reablement in Norway: a clinical controlled trial. BMC geriatrics. 2019;19(1):29. 
14. Lewin G, Calver J, McCormack B, al. e. The Home Independence Project. Geriaction. 2008;26(3):13-20.

15. Lewin GF, Alfonso HS, Alan JJ. Evidence for the long term cost effectiveness of home care reablement programs. Clin Interv Aging. 2013;8:1273-1281.

16. Lewin GF, Vandermeulen S. A non-randomised controlled trial of the Home Independence Program (HIP): an Australian restorative programme for older home-care clients. Health 85 Social Care in the Community.2010;18(1):91-99.

17. Sims-Gould J, Tong CE, Wallis-Mayer L, Ashe MC. Reablement, reactivation, rehabilitation and restorative interventions with older adults in receipt of home care: a systematic review. Journal of the American Medical Directors Association. 2017;18(8):653-663.

18. Shapiro A, Taylor M. Effects of a community-based early intervention program on the subjective wellbeing, institutionalization, and mortality of low-income elders. Gerontologist.2002;42(3):334-341.

19. Scharlach AE, Graham CL, Berridge C. An integrated model of co-ordinated community-based care. The Gerontologist.2015;55(4):677-687.

20. Szanton SL, Leff B, Wolff JL, Roberts L, Gitlin LN. Home-Based Care Program Reduces Disability And Promotes Aging In Place. Health Affairs (Project Hope). 2016;35(9):1558-1563.

21. Parsons M, Senior HEJ, Kerse N, et al. The Assessment of Services Promoting Independence and Recovery in Elders Trial (ASPIRE): a pre-planned meta-analysis of three independent randomised controlled trial evaluations of ageing in place initiatives in New Zealand.Age And Ageing. 2012;41(6):722-728.

22. Tappenden P, Campbell F, Rawdin A, Wong R, Kalita N. The clinical effectiveness and cost-effectiveness of home-based, nurse-led health promotion for older people: a systematic review. Health Technology Assessment (Winchester, England). 2012;16(20):1-72.

23. Fagerstrom L, Wikblad A, Nilsson J. An integrative research review of preventive home visits among older people-is an individual health resource perspective a vision or a reality? Scandinavian journal of caring sciences. 2009;23(3):558-568.

24. Bouman A, van Rossum E, Nelemans P, Kempen GI, Knipschild P. Effects of intensive home visiting programs for older people with poor health status: a systematic review. BMC Health Serv Res. 2008;8:74.

25. Berger S, Escher A, Mengle E, Sullivan N. Effectiveness of Health Promotion, Management, and Maintenance Interventions Within the Scope of Occupational Therapy for CommunityDwelling Older Adults: A Systematic Review. AMERICAN JOURNAL OF OCCUPATIONAL THERAPY.2018;72(4):7204190010p7204190011.

26. Sheffield C, Smith CA, Becker M. Evaluation of an Agency-Based Occupational Therapy Intervention to Facilitate Aging in Place.GERONTOLOGIST. 2013;53(6):907-918.

27. King AI, Parsons M, Robinson E, Jorgensen D. Assessing the impact of a restorative home care service in New Zealand: a cluster randomised controlled trial. Health Soc Care Community. 2012;20(4):365-374.

28. Parsons M, Senior H, Kerse N, Chen MH, Jacobs S, Anderson C. Randomised trial of restorative home care for frail older people in New Zealand. Nurs Older People. 2017;29(7):27-33.

29. Luker JA, Worley A, Stanley M, Uy J, Watt AM, Hillier SL. The evidence for services to avoid or delay residential aged care admission: a systematic review. BMC geriatrics. 2019;19(1):217.

30. Hammar T, Perala ML, Rissanen P. The effects of integrated home care and discharge practice on functional ability and health-related quality of life: a cluster-randomised trial among home care patients. Int J Integr Care. 2007;7:e29. 
31. Spoorenberg SL, Wynia K, Uittenbroek RJ, Kremer HP, Reijneveld SA. Effects of a population-based, person-centred and integrated care service on health, wellbeing and self-management of community-living older adults: A randomised controlled trial on Embrace. PloS one.2018;13(1):e0190751.

32. Beland F, Bergman H, Lebel P, et al. A system of integrated care for older persons with disabilities in Canada: results from a randomized controlled trial. J Gerontol A Biol Sci Med Sci.2006;61(4):367-373.

33. Markle-Reid M, Browne G, Gafni A. Nurse-led health promotion interventions improve quality of life in frail older home care clients: lessons learned from three randomized trials in Ontario, Canada.Journal of Evaluation in Clinical Practice. 2013;19(1):118-131.

34. Suijker JJ, van Rijn M, Buurman BM, Ter Riet G, Moll van Charante EP, de Rooij SE. Effects of NurseLed Multifactorial Care to Prevent Disability in Community-Living Older People: Cluster Randomized Trial.Plos One. 2016;11(7):e0158714-e0158714.

35. Pearson S, Inglis SC, McLennan SN, et al. Prolonged Effects of a Home-Based Intervention in Patients With Chronic Illness. Archives of internal medicine (1960). 2006;166(6):645-650.

36. Stuck AE, Egger M, Hammer A, Minder CE, Beck JC. Home visits to prevent nursing home admission and functional decline in elderly people: systematic review and meta-regression analysis. JAMA.2002;287(8):1022-1028.

37. van Rossum E, Frederiks CM, Philipsen H, Portengen K, Wiskerke J, Knipschild P. Effects of preventive home visits to elderly people. BMJ. 1993;307(6895):27-32.

38. Fletcher K, Mant J. A before and after study of the impact of Specialist Workers for Older People. Journal of evaluation in clinical practice. 2009;15(2):335-340.

39. Gagnon AJ, Schein C, McVey L, Bergman H. Randomized controlled trial of nurse case management of frail older people. JOURNAL OF THE AMERICAN GERIATRICS SOCIETY. 1999;47(9):1118-1124.

40. Godwin M, Gadag V, Pike A, et al. A randomized controlled trial of the effect of an intensive 1-year care management program on measures of health status in independent, community-living old elderly: the Eldercare project. Family Practice. 2016;33(1):37-41.

41. Imhof L, Naef R, Wallhagen MI, Schwarz J, Mahrer-Imhof R. Effects of an Advanced Practice Nurse In-Home Health Consultation Program for Community-Dwelling Persons Aged 80 and Older. Journal of the American Geriatrics Society (JAGS). 2012;60(12):2223-2231.

42. Harris R, Ashton T, Broad J, Connolly G, Richmond D. The effectiveness, acceptability and costs of a hospital-at-home service compared with acute hospital care: a randomized controlled trial.Journal Of Health Services Research 83 Policy.2005;10(3):158-166.

43. Low L-F, Fletcher J, Gresham M, Brodaty H. Community care for the Elderly: Needs and Service Use Study (CENSUS): Who receives home care packages and what are the outcomes? Australasian Journal On Ageing. 2015;34(3):E1-E8.

44. Bernabei R, Landi F, Gambassi G, et al. Randomised trial of impact of model of integrated care and case management for older people living in the community. BMJ. 1998;316(7141):1348-1351.

45. Landi F, Gambassi G, Pola R, et al. Impact of Integrated Home Care Services on Hospital Use. Journal of the American Geriatrics Society. 1999;47(12):1430-1434.

46. Beland F, Bergman H, Lebel P, et al. Integrated services for frail elders (SIPA): a trial of a model for Canada. Can J Aging.2006;25(1):5-42.

47. Stewart MJ, Georgiou A, Westbrook JI. Successfully integrating aged care services: a review of the evidence and tools emerging from a long-term care program. International Journal of Integrated Care.2013;13. 
48. Onder G, Liperoti R, Soldato M, et al. Case management and risk of nursing home admission for older adults in home care: results of the AgeD in HOme Care Study. Journal of the American Geriatrics Society. 2007;55(3):439-444.

49. Markle-Reid M, Weir R, Browne G, Roberts J, Gafni A, Henderson S. Health promotion for frail older home care clients. Journal of Advanced Nursing. 2006;54(3):381-395.

50. Morales-Asencio JM, Gonzalo-Jimenez E, Martin-Santos FJ, et al. Effectiveness of a nurse-led case management home care model in Primary Health Care. A quasi-experimental, controlled, multi-centre study.BMC Health Services Research. 2008;8:193-193.

51. Bass DM, Judge KS, Lynn Snow A, et al. Caregiver outcomes of partners in dementia care: effect of a care coordination program for veterans with dementia and their family members and friends. Journal of the American Geriatrics Society. 2013;61(8):1377-1386.

52. Haggerty JL, Pineault R, Beaulieu M-D, et al. Practice Features Associated With Patient- Reported Accessibility, Continuity, and Coordination of Primary Health Care. Annals of Family Medicine.2008;6(2):116123.

53. Santomassino M, Costantini GD, McDermott M, Primiano D, Slyer JT, Singleton JK. A systematic review on the effectiveness of continuity of care and its role in patient satisfaction and decreased hospital readmissions in the adult patient receiving home care services.JBI database of systematic reviews and implementation reports.2012;10(21):1214-1259.

54. Russell D, Rosati RJ, Rosenfeld P, Marren JM. Continuity in home health care: is consistency in nursing personnel associated with better patient outcomes? Journal For Healthcare Quality: Official Publication Of The National Association For Healthcare Quality.2011;33(6):33-39.

55. Goodman C, Drennan V, Manthorpe J, et al. A study of the effectiveness of inter-professional working for community dwelling older people: Final report-NIHR Service Delivery \& Organisation Programme, 2011. NIHR Final Report. 2012.

56. Kodner DL. Whole-system approaches to health and social care partnerships for the frail elderly: an exploration of North American models and lessons. Health 85 Social Care in the Community.2006;14(5):384390.

57. Mintzberg H. Time for the Plural Sector. Stanford Social Innovation Review. 2015(Summer):28-33.

58. Henderson EJ, Caplan GA. Home sweet home? Community care for older people in Australia. Journal Of The American Medical Directors Association. 2008;9(2):88-94.

59. Leichsenring K. Integrated care for older people in Europe - latest trends and perceptions. International Journal of Integrated Care.2012;12(30):1-4.

60. Calabresi G, Bobbitt P. Tragic Choices. New-York: W.W. Norton \& Company; 1978.

61. Boling PA, Chandekar RV, Hungate B, Purvis M, Selby-Penczak R, Abbey LJ. Improving outcomes and lowering costs by applying advanced models of in-home care. Cleve Clin J Med. 2013;80 Electronic Suppl 1:eS7-14.

62. Duner A. Care planning and decision-making in teams in Swedish elderly care: a study of interprofessional collaboration and professional boundaries. Journal Of Interprofessional Care.2013;27(3):246-253.

63. Johri M, Beland F, Bergman H. International experiments in integrated care for the elderly: a synthesis of the evidence. Int J Geriatr Psychiatry. 2003;18(3):222-235.

64. Brown L, Tucker C, Domokos T. Evaluating the impact of integrated health and social care teams on older people living in the community. Health Soc Care Community. 2003;11(2):85-94. 
65. Low L-F, Yap M, Brodaty H. A systematic review of different models of home and community care services for older persons. BMC Health Services Research. 2011;11:93-93.

66. Shortell SM, Gillies RR, Anderson DA. The New World of Managed Care: Creating Organized Delivery Systems. Health Affairs.1994;13(5):46-64.

67. Shortell SM, Gillies RR, Devers KJ. Reinventing the American Hospital. The Milbank Quarterly. 1995;73(2):131-160.

68. Kodner D. All Together Now: A Conceptual Exploration of Integrated Care. Healthcare Quarterly. 2009;13(Sp):6-15.

69. Weinberg DB, Lusenhop RW, Gittell JH, Kautz CM. Coordination between formal providers and informal caregivers. Health Care Management Review. 2007;32(2):140-149.

70. Balinsky W, LaPolla JF. The New York City shared aide program (cluster care): a model for the future. Home Health Care Services Quarterly. 1993;14(1):41-54.

71. Gray YL, Sedhom L. Client satisfaction: traditional care versus cluster care. Journal of Professional Nursing. 1997;13(1):56-61.

72. Bjornsdottir K. 'I try to make a net around each patient': home care nursing as relational practice. Scandinavian journal of caring sciences. 2018;32(1):177-185.

73. Mahase E. Mortality rates are lower with higher continuity of care, review finds. BMJ. 2020;370:m3184.

74. Pereira Gray DJ, Sidaway-Lee K, White E, Thorne A, Evans PH. Continuity of care with doctors-a matter of life and death? A systematic review of continuity of care and mortality. BMJ Open.2018;8(6):e021161e021161.

Acknowledgements: Special thanks to all of our coders, including Cynthia Yamamoto, Alexandra Ethier, Melissa Giesbrecht, Breanna Horne and to the rest of our project team. Annie Carrier is a Fonds de recherche du Quebec - Sante Junior 1 researcher (\#296437).

Conflict of Interest statement : The authors report having no conflicts of interest.

Funding: This work was funded by the Canadian Institutes for Health Research (Grant \# 162278)

Figure legend: Figure 1: Search process flowchart 
figures/Figure1/Figure1-eps-converted-to.pdf 